\title{
ARTIGO ORIGINAL Interações familiares de estudantes em situações de bullying
}

\author{
Family interactions of students in bullying situations
}

Wanderlei Abadio de Oliveira', Jorge Luiz da Silva², Manoel Antônio dos Santos', Miyeko Hayashidaª, Simona Carla Silvia Caravita ${ }^{4}$, Marta Angélica lossi Silva ${ }^{3}$

\section{RESUMO}

Objetivo: Analisar a qualidade das interações familiares de adolescentes e o envolvimento em situações de bullying escolar. Métodos: Estudo transversal de base populacional que contou com a participação de 2.354 estudantes (meninas = 50,7\%; idade média $M=14,5$ anos, DP = 2,0 anos) do ensino fundamental e médio, de 11 escolas públicas de uma cidade do interior de Minas Gerais. A amostra do estudo foi definida pela seleção de estratos (escolas), utilizando o método Probability Proportional to Size. A coleta de dados ocorreu por meio da aplicação coletiva de duas escalas, sendo uma para identificar comportamentos de bullying/vitimização e outra para aferir a qualidade das interações familiares dos adolescentes. Os procedimentos de análise incluíram: análise estatística descritiva, análise de variância (ANOVA) e regressão logística. Resultados: O estudo identificou uma prevalência de 10,3\% de estudantes agressores, 10,1\% de vítimas e 5,4\% de vítimas-agressoras na amostra. Verificou-se que os estudantes não envolvidos em situações de bullying possuíam melhores interações familiares quando comparados com os estudantes identificados como agressores, vítimas e vítimas-agressoras. Efeitos do aspecto "regras e monitoria" (OR: 1,21; $p=0,001$ ) no contexto familiar foram identificados como protetivo para o bullying ou a vitimização. Os aspectos "punição física" (OR: 0,84; $p=0,001)$ e "comunicação negativa" (OR: 0,53; $p=0,001)$ foram associados a maior possibilidade de bullying ou vitimização entre os estudantes. Conclusões: Confirmou-se que a qualidade das interações familiares influencia no envolvimento dos estudantes em situações de bullying. Implicações para a área da saúde foram discutidas a partir de indicadores de risco e proteção identificados.

\section{ABSTRACT}

Objective: To analyze the quality of the family interactions of adolescents and the involvement in situations of school bullying. Methods: A population-based cross-sectional study involving 2,354 students (girls $=50.7 \%$, mean age $M=14.5$ years, $S D=2.0$ years) of 11 primary schools in a city of the interior of Minas Gerais. The study sample was defined by stratum selection (schools), using the Probability Proportional to Size method. The data collection occurred through collective application of two scales, one to identify behaviors of bullying/victimization and another to measure the quality of family interactions of the adolescents. The analysis

1 Universidade de São Paulo (USP), Faculdade de Filosofia, Ciências e Letras de Ribeirão Preto, Ribeirão Preto, SP, Brasil. 2 Universidade de Franca (Unifran), Programa de Pós-Graduação em Promoção da Saúde, Franca, SP, Brasil. 3 Universidade de São Paulo (USP), Escola de Enfermagem de Ribeirão Preto, Ribeirão Preto, SP, Brasil. 


\section{Keywords}

School health, mental health, adolescente health, violence, risk-taking. procedures included: descriptive statistical analysis, analysis of variance (ANOVA) and logistic regression. Results: The study found a prevalence of $10.3 \%$ of the aggressors students, $10.1 \%$ of the victims and $5.4 \%$ of the victims-aggressors in the sample. It was found that students not involved in bullying situations had better family interactions when compared to students identified as aggressors, victims and victims-aggressors. Effects of the "rules and monitoring" aspect (OR: 1,21; $p=0,001)$ in the family context were identified as protective for bullying or victimization. The "physical punishment" (OR: 0,84, $p=0,001)$ and "negative communication" (OR: 0,53; $p=0,001$ ) aspects were associated with a greater possibility of bullying or victimization among the students. Conclusions: It was confirmed that the quality of family interactions influences the involvement of the students in bullying situations. Implications for the health area were discussed from identified risk and protection indicators.

\section{INTRODUÇÃO}

O bullying em diferentes contextos é considerado um problema de saúde pública ${ }^{1,2}$, caracterizado pela intencionalidade e pela repetitividade das agressões, bem como pelo desequilíbrio de poder existente entre vítimas e agressores $^{3}$. Seus efeitos na saúde mental de crianças e adolescentes já foram documentados pela literatura científica e se referem a problemas de adaptação e ajuste psicoemocional, adoecimentos psicossomáticos e psicológicos, quadros e sintomatologia depressiva, ideação e tentativas de suicídio ${ }^{1,3}$. No Brasil, o fenômeno tem recebido crescente atenção nas últimas décadas e se identifica um aumento no número de estudos divulgados sobre as características pessoais dos estudantes envolvidos em situações de bullying, diagnósticos e aspectos relacionados ao contexto escolar ${ }^{4}$. No entanto, a abordagem do fenômeno no país ainda é centrada exclusivamente na díade agressor/vítima, com poucos estudos que incluem os observadores em suas análises, ou no cenário escolar e seus aspectos que podem ser utilizados para a explicar sua ocorrência.

Estudos internacionais indicam que aspectos familiares devem ser considerados na análise do bullying ${ }^{5}$. Variáveis familiares, tais como tipo de envolvimento, clima afetivo, uso de punições físicas, conflitos, relacionamentos negativos, falta de supervisão e dificuldades de comunicação, são apontadas pela literatura como preditoras ou fatores de vulnerabilidade para experiências como agressores ou vítimas em situações de bullying ${ }^{6-9}$. Em termos de revisão, destacam-se dois estudos brasileiros que investigaram a associação entre a expressão da violência no ambiente escolar e a punição física utilizada como medida de disciplina no contexto familiar ${ }^{10}$ e a presença de pais negligentes, agressivos, em conflito, que empreendem maus-tratos físicos e emocionais aos filhos ${ }^{11}$.

Entende-se, nesse sentido, que o planejamento e o desenvolvimento das ações de enfrentamento da questão precisam considerar outras variáveis e serem implementados de forma intersetorial. Esse debate representa uma mudança paradigmática para a área da saúde, tanto no que se refere ao reconhecimento do bullying como problema de saúde, como na percepção de que os estudantes vítimas ou agressores somente serão mais saudáveis quando os planos de cuidado não se basearem na culpabilização individual, mas na compreensão de que a saúde possui determinantes e condicionantes, assim como os fenômenos sociais ${ }^{5-7}$. Uma iniciativa política intersetorial nessa direção se refere ao Programa Saúde na Escola12, que estimula a arquitetura de ações a partir das novas compreensões sobre a promoção da saúde nas instituições escolares, incluindo o bullying como temática transversal a ser abordada pela área na atenção primária e parceria com outras áreas, sobretudo a educação.

A partir desse cenário, estudar as implicações da família na ocorrência do bullying escolar ou como fator que pode auxiliar em sua explicação pode contribuir para: 1) propiciar comparação com estudos desenvolvidos em outros contextos e 2) na proposição de intervenções contextuais e intersetoriais que incluam a família, principalmente no contexto da atenção primária à saúde. Assim sendo, e considerando a produção científica limitada no país e na área da saúde sobre a interface entre bullying e variáveis familiares, esse estudo objetivou analisar a qualidade das interações familiares de adolescentes e o envolvimento em situações de bullying escolar.

\section{MÉTODOS}

\section{Participantes}

O estudo foi desenvolvido em 11 escolas de um município do interior de Minas Gerais, Brasil. A amostra probabilística foi definida pela seleção de estratos (escolas), utilizando o método Probability Proportional to Size ${ }^{13}$. Todos os estudantes ( $N=2.729$ ) do ensino fundamental e médio das instituições selecionadas foram convidados para participar e, por fim, participaram do estudo 2.354 estudantes com idades entre 10 e 19 anos (meninas = 50,7\%; idade média $M=14,5$ anos, $\mathrm{DP}=2,0$ anos). 


\section{Procedimentos}

A coleta de dados ocorreu durante uma semana em cada uma das 11 escolas e durante o horário das aulas, entre os meses de agosto e outubro de 2014. No primeiro contato com os estudantes se apresentavam os objetivos, os procedimentos do estudo e todos eram convidados a participar da pesquisa. Após recolhimento dos termos de consentimento e assentimento, os estudantes respondiam a "Escala de Vitimização e Agressão entre Pares" (EVAP) ${ }^{14}$ e a "Escala de Qualidade na Interação Familiar" (EQIF) ${ }^{15}$. A aplicação das escalas foi coletiva e durou, em média, 50 minutos. Os dados das escalas foram inseridos em planilha Excel, com dupla digitação, e uso de procedimento de validação. Por fim, procedeu-se a análise dos dados no software Statistical Package for the Social Sciences.

\section{Instrumentos e técnica de pesquisa}

\section{Escala de Agressão e Vitimização entre Pares (EVAP)}

Instrumento de autorrelato validado para o contexto brasileiro, publicado e desenvolvido para investigar a agressão entre pares no contexto escolar. A escala contém 18 questões afirmativas (eu provoquei colegas; eu xinguei colegas; colegas roubaram, mexeram ou estragaram minhas coisas, por exemplo) que são avaliadas em sistema Likert de cinco pontos para medir a frequência dos comportamentos (1 nunca; 2 - quase nunca; 3 - às vezes; 4 - sempre; 5 - quase sempre) ${ }^{14}$. Por meio dos resultados dessa escala, os participantes foram divididos em quatro grupos: 1) agressores; 2) vítimas; 3) vítimas-agressoras; 4) não envolvidos. A estrutura da escala, em relação ao grupo amostral, foi testada por meio de análise fatorial e verificada pelos testes $\mathrm{KMO}(0,90$ e 0,89$)$ e Bartlett's Test $(p=0,000)$. Nessa análise, os 10 itens de avaliação da agressividade dos estudantes alcançaram índice de consistência interna no valor 0,83, e os nove itens da escala de vitimização apresentaram alfa de Cronbach no valor de 0,84 .

\section{Escala de Qualidade de Interação Familiar (EQIF)}

Instrumento validado para o contexto brasileiro, publicado e que avalia aspectos do relacionamento da família por meio do relato de crianças ou adolescentes acerca da interação com os pais, abordando nove fatores da interação familiar ${ }^{15}$ : 1. Envolvimento que inclui características das relações entre os membros do grupo familiar; 2 . Regras e monitoria, relacionada à existência de normas e regras que devem ser observadas pelos filhos, bem como a supervisão parental; 3 . Punição corporal ou o uso de técnicas de disciplina baseadas na agressão física; 4. Comunicação positiva; 5. Comunicação negativa; 6. Clima conjugal positivo; 7. Clima conjugal negativo; 8. Modelo parental, dimensão que verifica se os pais são exemplos positivos para os filhos; e 9. Sentimento dos filhos em relação aos pais. $O$ instrumento é composto por 40 itens, mensurados por meio de um sistema Likert de cinco pontos ( 1 - nunca; 2 - quase nunca; 3 - às vezes; 4 - sempre; 5 - quase sempre). São exemplos de questões afirmativas da escala: meus pais fazem carinho um no outro; meus pais costumam me xingar ou falar palavrões para mim etc. A estrutura da escala, em relação ao grupo amostral, foi testada por meio de análise fatorial e verificada pelos testes $\mathrm{KMO}(0,86 \mathrm{e}$ $0,60)$ e Bartlett's Test $(p=0,000)$. Os índices de consistência interna das nove dimensões avaliadas nas duas escalas atingiram 0,65 (aspectos negativos) e 0,84 (aspectos positivos), apresentando índices satisfatórios de confiabilidade para o tamanho amostral e o tipo de estudo desenvolvido (exploratório e transversal).

\section{Análise dos dados}

Os dados foram digitados no programa Excel e, posteriormente, importados para o software Statistical Package for the Social Sciences, versão 21.0. Optou-se pela recomendação teórica e se presumiu a distribuição normal das variáveis considerando o grande conjunto de dados. Inicialmente, foi realizada uma exploração descritiva e de correlação dos dados. Em seguida, as diferenças e igualdades entre as médias dos grupos de envolvimento em situações de bullying em relação às nove dimensões da qualidade de interação familiar foram verificadas por meio da análise de variância ANOVA e o post hoc de Turkey (Z). Fatores preditores relacionados à interação familiar para o bullying/vitimização foram analisados por meio de modelos de regressão logística. Para a análise estatística, considerou-se o nível de significância a = 0,05.

\section{Questões éticas}

Todas as fases do estudo seguiram as orientações, exigências e recomendações da Resolução 466/2012. O projeto foi submetido e aprovado pelo Comitê de Ética em Pesquisa da Escola de Enfermagem de Ribeirão Preto da Universidade de São Paulo (EERP/USP) (Parecer: 484.912/2013).

\section{RESULTADOS}

A taxa de participação no estudo foi de $74,5 \%$ (considerando o número total de estudantes matriculados no turno matutino nas 11 escolas selecionadas) e 2.354 adolescentes concluíram o preenchimento das duas escalas utilizadas na coleta de dados. Para investigar as categorias de envolvimento em agressão (bullying) e vitimização entre os estudantes, foi utilizada a análise de agrupamento K-means clustering. Os participantes foram, então, divididos em quatro grupos (clusters), dos quais $74,2 \%$ se concentraram no grupo sem nenhum tipo de envolvimento com as situações de bullying, 10,3\% foram identificados como agressores, 10,1\% como vítimas e 5,4 como vítimas-agressoras. Na Tabela 1 estão apresentadas, de forma detalhada, as características da amostra do es- 
tudo segundo os quatro clusters identificados: o sexo, a idade, o ano escolar e a configuração familiar dos participantes.

Cálculos de Pearson constataram correlações estatisticamente significantes entre a idade $(p=0,001)$ e ano escolar $(p=0,001)$ com a vitimização, indicando que quanto mais novo e inserido nas séries iniciais, maior a chance de o participante ser vítima de bullying. Para os outros grupos não foram encontradas diferenças estatisticamente significantes na análise dessas variáveis. No que se refere aos arranjos familiares dos participantes a partir dessa divisão, verificou-se que se sobressaíram, nos quatro grupos, os arranjos nucleares, nos quais as duas figuras parentais estavam presentes.
Pela análise de variância (ANOVA), foram verificadas diferenças estatísticas significativas $(p<0,005)$ entre os quatro grupos de estudantes no que se refere à qualidade da interação familiar, e nas comparações post hoc (Z) entre elas. Na Tabela 2, são apresentados os dados da ANOVA em relação às dimensões da qualidade da interação familiar geral dos adolescentes e o tipo de envolvimento em situações de bullying.

Observa-se que os estudantes não envolvidos com as situações de bullying apresentaram maiores médias em todas as escalas positivas (envolvimento, regras e monitoria, comunicação positiva, clima conjugal positivo, modelo e

Tabela 1. Caracterização dos estudantes $(N=2.354)$ segundo o tipo de envolvimento em situações de bullying e por sexo

\begin{tabular}{|c|c|c|c|c|c|c|c|c|c|c|c|c|c|c|c|c|c|c|}
\hline & \multicolumn{2}{|c|}{ Total } & \multicolumn{4}{|c|}{ Agressor } & \multicolumn{4}{|c|}{ Vítima } & \multicolumn{4}{|c|}{ Vitima-agressora } & \multicolumn{4}{|c|}{ Não envolvimento } \\
\hline & \multirow{2}{*}{$\mathrm{N}$} & \multirow{2}{*}{$\%$} & \multicolumn{2}{|c|}{ Masculino } & \multicolumn{2}{|c|}{ Feminino } & \multicolumn{2}{|c|}{ Masculino } & \multicolumn{2}{|c|}{ Feminino } & \multicolumn{2}{|c|}{ Masculino } & \multicolumn{2}{|c|}{ Feminino } & \multicolumn{2}{|c|}{ Masculino } & \multicolumn{2}{|c|}{ Feminino } \\
\hline & & & $n$ & $\%$ & $\mathrm{n}$ & $\%$ & $\mathrm{n}$ & $\%$ & $\mathrm{n}$ & $\%$ & $\mathrm{n}$ & $\%$ & $n$ & $\%$ & $\mathrm{n}$ & $\%$ & $n$ & $\%$ \\
\hline \multicolumn{19}{|l|}{ Idade } \\
\hline 10 & 15 & 0,70 & 2 & 0,10 & 0 & 0,00 & 3 & 0,15 & 0 & 0,00 & 2 & 0,10 & 0 & 0,00 & 5 & 0,20 & 3 & 0,15 \\
\hline 11 & 186 & 7,90 & 1 & 0,05 & 0 & 0,00 & 17 & 0,75 & 14 & 0,60 & 4 & 0,15 & 3 & 0,15 & 68 & 2,95 & 79 & 3,30 \\
\hline 12 & 262 & 11,10 & 11 & 0,45 & 5 & 0,20 & 18 & 0,75 & 12 & 0,50 & 15 & 0,65 & 2 & 0,10 & 99 & 4,25 & 100 & 4,20 \\
\hline 13 & 306 & 13,00 & 20 & 0,85 & 7 & 0,30 & 25 & 1,10 & 17 & 0,70 & 9 & 0,40 & 10 & 0,40 & 102 & 4,30 & 116 & 4,85 \\
\hline 14 & 361 & 15,30 & 39 & 1,70 & 19 & 0,80 & 20 & 0,85 & 25 & 1,05 & 7 & 0,30 & 7 & 0,30 & 110 & 4,50 & 134 & 5,60 \\
\hline 15 & 387 & 16,40 & 42 & 1,80 & 14 & 0,60 & 11 & 0,45 & 18 & 0,75 & 18 & 0,75 & 3 & 0,15 & 121 & 5,20 & 160 & 6,70 \\
\hline 16 & 403 & 17,10 & 30 & 1,30 & 9 & 0,40 & 11 & 0,45 & 21 & 0,90 & 14 & 0,60 & 8 & 0,35 & 136 & 5,50 & 174 & 7,40 \\
\hline 17 & 320 & 13,60 & 24 & 1,05 & 13 & 0,55 & 7 & 0,30 & 11 & 0,45 & 18 & 0,75 & 2 & 0,10 & 101 & 4,35 & 144 & 6,10 \\
\hline 18 & 98 & 4,20 & 5 & 0,20 & 1 & 0,05 & 2 & 0,10 & 3 & 0,15 & 5 & 0,20 & 0 & 0,00 & 31 & 1,35 & 51 & 2,15 \\
\hline 19 & 16 & 0,70 & 0 & 0,00 & 1 & 0,05 & 1 & 0,05 & 1 & 0,05 & 0 & 0,00 & 0 & 0,00 & 8 & 0,35 & 5 & 0,20 \\
\hline \multicolumn{19}{|l|}{ Ano escolar } \\
\hline $\begin{array}{l}6^{\circ} \text { ano Ensino } \\
\text { Fundamental }\end{array}$ & 332 & 14,10 & 10 & 0,45 & 1 & 0,05 & 35 & 1,50 & 21 & 0,90 & 18 & 0,75 & 6 & 0,25 & 126 & 5,20 & 115 & 4,85 \\
\hline $\begin{array}{l}70 \text { ano Ensino } \\
\text { Fundamental }\end{array}$ & 307 & 13,05 & 29 & 1,25 & 9 & 0,40 & 12 & 0,50 & 20 & 0,85 & 14 & 0,60 & 4 & 0,15 & 115 & 4,95 & 104 & 4,45 \\
\hline $\begin{array}{l}8^{\circ} \text { ano Ensino } \\
\text { Fundamental }\end{array}$ & 362 & 15,45 & 41 & 1,75 & 15 & 0,65 & 30 & 1,30 & 13 & 0,55 & 10 & 0,45 & 8 & 0,35 & 111 & 4,80 & 134 & 5,60 \\
\hline $\begin{array}{l}90 \text { ano Ensino } \\
\text { Fundamental }\end{array}$ & 387 & 16,45 & 30 & 1,30 & 17 & 0,70 & 16 & 0,70 & 28 & 1,15 & 12 & 0,50 & 8 & 0,35 & 125 & 5,20 & 151 & 6,40 \\
\hline $1^{\circ}$ ano Ensino Médio & 389 & 16,50 & 29 & 1,25 & 13 & 0,55 & 11 & 0,45 & 18 & 0,75 & 17 & 0,75 & 4 & 0,15 & 133 & 5,40 & 164 & 7,00 \\
\hline $2^{\circ}$ ano Ensino Médio & 337 & 14,25 & 23 & 1,00 & 6 & 0,25 & 9 & 0,40 & 16 & 0,65 & 13 & 0,55 & 4 & 0,15 & 106 & 4,55 & 160 & 6,70 \\
\hline $3^{0}$ ano Ensino Médio & 240 & 10,20 & 12 & 0,50 & 8 & 0,35 & 2 & 0,10 & 6 & 0,25 & 8 & 0,35 & 1 & 0,05 & 65 & 2,80 & 138 & 5,80 \\
\hline \multicolumn{19}{|l|}{ Arranjo familiar } \\
\hline Pai e mãe & 598 & 25,70 & 49 & 2,05 & 15 & 0,35 & 32 & 1,35 & 14 & 0,60 & 23 & 1,00 & 9 & 0,40 & 238 & 10,00 & 218 & 9,25 \\
\hline Pai, mãe e irmão(s) & 638 & 27,15 & 46 & 1,95 & 18 & 0,75 & 28 & 1,15 & 26 & 1,05 & 19 & 0,80 & 8 & 0,35 & 193 & 8,40 & 300 & 12,80 \\
\hline $\begin{array}{l}\text { Pai, mãe, irmão(s) e/ou } \\
\text { outras pessoas }\end{array}$ & 110 & 4,70 & 6 & 0,25 & 4 & 0,15 & 8 & 0,35 & 9 & 0,40 & 5 & 0,20 & 1 & 0,05 & 42 & 1,80 & 35 & 1,50 \\
\hline Somente com o pai & 43 & 1,90 & 8 & 0,35 & 0 & 0,00 & 3 & 0,15 & 1 & 0,05 & 2 & 0,10 & 0 & 0,00 & 14 & 0,60 & 15 & 0,65 \\
\hline Somente com a mãe & 263 & 11,05 & 23 & 1,00 & 5 & 0,20 & 16 & 0,65 & 20 & 0,85 & 18 & 0,75 & 2 & 0,10 & 92 & 3,85 & 87 & 3,65 \\
\hline Pai e outras pessoas & 67 & 2,85 & 5 & 0,20 & 2 & 0,10 & 7 & 0,30 & 1 & 0,05 & 3 & 0,15 & 1 & 0,05 & 22 & 0,95 & 26 & 1,05 \\
\hline Mãe e outras pessoas & 513 & 21,50 & 30 & 1,30 & 21 & 0,90 & 19 & 0,80 & 40 & 1,70 & 12 & 0,50 & 12 & 0,50 & 132 & 5,55 & 247 & 10,45 \\
\hline Outras pessoas & 122 & 5,15 & 7 & 0,30 & 4 & 0,15 & 2 & 0,10 & 11 & 0,45 & 10 & 0,40 & 2 & 0,10 & 48 & 2,00 & 38 & 1,65 \\
\hline
\end{tabular}


sentimento dos filhos), diferenciando-se dos demais grupos. Nas escalas negativas (punição física, comunicação negativa e clima conjugal negativo), observa-se que agressores, vítimas e vítimas-agressoras apresentaram maior média em relação aos estudantes não envolvidos. Na análise da qualidade da interação familiar geral, as vítimas e as vítimas-agressoras se diferenciaram significativamente dos outros dois grupos no que se refere à "punição física". A "comunicação negativa" dos agressores, vítimas e vítimas-agressoras apresentou diferença significativa em relação ao grupo dos estudantes não envolvidos. O mesmo ocorreu com a dimensão "clima conjugal negativo".

A partir desses resultados, a comparação entre os quatro grupos de estudantes identificados pelo estudo (variáveis dependentes) em relação ao sexo, à idade e às noves dimensões de qualidade da interação familiar (variáveis independentes), por meio da regressão logística, permitiu a identificação de algumas diferenças estatisticamente significantes entre os grupos (Tabela 3).

As dimensões "comunicação negativa" (OR: 1,69; $p=$ 0,001) e "clima conjugal negativo" (OR: 1,$17 ; p=0,002$ ) aumentavam as chances de os estudantes serem inseridos no grupo de agressores. As dimensões "punição física" (OR: 1,26; $p=0,001$ ) e "comunicação negativa" (OR: 1,81; $p=0,001)$ se mantiveram associadas significativamente com a condição de vítimas dos estudantes, indicando que a ocorrência de situações dessa natureza no ambiente doméstico aumentava a chance de vitimização. A dimensão

Tabela 2. ANOVA de acordo com o agrupamento dos estudantes $(N=2.354)$ e em relação à qualidade da interação familiar geral

\begin{tabular}{|c|c|c|c|c|c|c|c|c|c|c|}
\hline \multirow{3}{*}{ Dimensōes - qualidade da interação familiar } & \multicolumn{8}{|c|}{ Clusters } & \multirow[b]{3}{*}{$z$} & \multirow[b]{3}{*}{$\mathrm{p}$} \\
\hline & \multicolumn{2}{|c|}{ Não envolvimento } & \multicolumn{2}{|c|}{ Agressores } & \multicolumn{2}{|c|}{ Vítimas } & \multicolumn{2}{|c|}{ Vítimas-agressoras } & & \\
\hline & M & DP & M & DP & M & DP & M & DP & & \\
\hline Envolvimento & $3,94 b$ & 0,89 & $3,62 a$ & 0,91 & $3,73 a$ & 1,04 & $3,71 a$ & 1,04 & 12,39 & 0,001 \\
\hline Regras e monitoria & $4,21 c$ & 0,78 & $3,85 a$ & 0,82 & $4,06 \mathrm{bc}$ & 0,87 & $4,00 \mathrm{ab}$ & 0,85 & 17,07 & 0,001 \\
\hline Punição física & $1,51 a$ & 0,74 & $1,72 b$ & 0,85 & $1,96 \mathrm{c}$ & 1,00 & $2,06 \mathrm{c}$ & 1,20 & 38,52 & 0,001 \\
\hline Comunicação positiva & $3,03 b$ & 1,11 & $2,66 a$ & 1,08 & $2,88 \mathrm{ab}$ & 1,15 & $2,82 a b$ & 1,07 & 9,35 & 0,001 \\
\hline Comunicação negativa & $2,02 a$ & 0,84 & $2,51 b$ & 0,92 & $2,54 b$ & 1,09 & $2,70 \mathrm{~b}$ & 1,09 & 56,24 & 0,001 \\
\hline Clima conjugal positivo & $3,22 b$ & 1,24 & $3,00 \mathrm{a}$ & 1,25 & $3,02 a b$ & 1,28 & $3,06 a b$ & 1,29 & 3,80 & 0,001 \\
\hline Clima conjugal negativo & $1,96 a$ & 0,92 & $2,33 b$ & 1,05 & $2,25 b$ & 1,12 & $2,42 b$ & 1,20 & 20,48 & 0,001 \\
\hline Modelo & $3,98 b$ & 0,89 & $3,73 a$ & 0,96 & $3,84 a b$ & 0,94 & $3,79 a b$ & 1,13 & 7,86 & 0,001 \\
\hline Sentimento dos filhos & $4,46 \mathrm{~b}$ & 0,74 & $4,24 a$ & 0,89 & $4,31 a b$ & 0,85 & $4,21 a$ & 1,06 & 10,42 & 0,001 \\
\hline
\end{tabular}

Notas: Nas linhas, as médias com letras iguais não se diferenciam entre si pelo pós-teste de Tukey $(p<0,005)$, sendo a $<\mathrm{b}<\mathrm{c}$.

M: média; DP: desvio-padrão.

Fonte: Elaborado pelos autores.

Tabela 3. Fatores da qualidade de interação familiar associados ao envolvimento ou não envolvimento de estudantes ( $\mathrm{N}=2.354)$ em situações de bullying

\begin{tabular}{|c|c|c|c|c|c|c|c|c|c|c|c|c|c|c|c|c|}
\hline & \multicolumn{4}{|c|}{ Agressores } & \multicolumn{4}{|c|}{ Vítimas } & \multicolumn{4}{|c|}{ Vitimas-agressoras } & \multicolumn{4}{|c|}{ Sem envolvimento } \\
\hline & \multirow{2}{*}{$\mathrm{OR}$} & \multicolumn{2}{|c|}{ IC $95 \%$} & \multirow{2}{*}{$p$} & \multirow{2}{*}{ OR } & \multicolumn{2}{|c|}{ IC $95 \%$} & \multirow{2}{*}{ p } & \multirow{2}{*}{ OR } & \multicolumn{2}{|c|}{ IC $95 \%$} & \multirow{2}{*}{$p$} & \multirow{2}{*}{ OR } & \multicolumn{2}{|c|}{ IC $95 \%$} & \multirow{2}{*}{$p$} \\
\hline & & L.I. & L.S. & & & L.I. & L.S. & & & L.I. & L.S. & & & L.I. & L.S. & \\
\hline Intercépito & 0,01 & & & 0,001 & 0,12 & & & 0,001 & 0,00 & & & 0,001 & 12,47 & & & 0,001 \\
\hline Sexo & 3,69 & 2,83 & 4,80 & 0,001 & 1,57 & 1,23 & 2,00 & 0,001 & 3,23 & 2,12 & 4,90 & 0,001 & 0,41 & 0,33 & 0,50 & 0,001 \\
\hline Idade & 1,05 & 0,99 & 1,12 & 0,110 & 0,87 & 0,81 & 0,92 & 0,001 & 1,00 & 0,91 & 1,11 & 0,960 & 1,07 & 1,01 & 1,12 & 0,002 \\
\hline Envolvimento & 1,07 & 0,84 & 1,35 & 0,580 & 1,05 & 0,83 & 1,33 & 0,680 & 1,27 & 0,87 & 1,84 & 0,210 & 0,98 & 0,80 & 1,19 & 0,083 \\
\hline Regras e monitoria & 0,80 & 0,67 & 0,94 & 0,001 & 0,97 & 0,81 & 1,15 & 0,700 & 0,98 & 0,75 & 1,29 & 0,900 & 1,21 & 1,04 & 1,40 & 0,001 \\
\hline Punição física & 1,09 & 0,94 & 1,26 & 0,270 & 1,26 & 1,09 & 1,45 & 0,001 & 1,22 & 0,99 & 1,51 & 0,006 & 0,84 & 0,74 & 0,95 & 0,001 \\
\hline Comunicação positiva & 0,92 & 0,80 & 1,05 & 0,200 & 1,00 & 0,88 & 1,14 & 0,980 & 0,96 & 0,78 & 1,18 & 0,710 & 1,05 & 0,94 & 1,17 & 0,400 \\
\hline Comunicação negativa & 1,69 & 1,43 & 2,01 & 0,001 & 1,81 & 1,52 & 2,15 & 0,001 & 1,80 & 1,38 & 2,34 & 0,001 & 0,53 & 0,46 & 0,62 & 0,001 \\
\hline Clima conjugal positivo & 1,04 & 0,93 & 1,16 & 0,530 & 0,97 & 0,87 & 1,08 & 0,570 & 1,01 & 0,85 & 1,21 & 0,910 & 1,01 & 0,92 & 1,10 & 0,920 \\
\hline Clima conjugal negativo & 1,17 & 1,02 & 1,33 & 0,002 & 1,01 & 0,89 & 1,16 & 0,820 & 1,08 & 0,88 & 1,33 & 0,440 & 0,91 & 0,81 & 1,02 & 0,009 \\
\hline Modelo & 0,97 & 0,80 & 1,16 & 0,710 & 1,03 & 0,85 & 1,24 & 0,770 & 0,99 & 0,74 & 1,32 & 0,930 & 1,00 & 0,85 & 1,17 & 0,960 \\
\hline Sentimento dos filhos & 1,14 & 0,90 & 1,44 & 0,290 & 1,09 & 0,86 & 1,39 & 0,460 & 0,92 & 0,64 & 1,32 & 0,660 & 0,84 & 0,68 & 1,03 & 0,009 \\
\hline
\end{tabular}

Fonte: Elaborado pelos autores. 
da qualidade de interação familiar "comunicação negativa" (OR: 1,80; $p=0,001$ ) se manteve associada significativamente com a condição de vítima-agressora. Por fim, a dimensão da qualidade de interação familiar "regras e monitoria" (OR: 1,21; $p=0,001)$ se apresentou com poder protetivo, na medida em que o aumento de sua pontuação diminuía a possibilidade de qualificação em um dos grupos de envolvimento com as situações de bullying. As dimensões "punição física" (OR: 0,84; $p=0,001)$ e "comunicação negativa" (OR: 0,53; $p=0,001)$ aumentavam a condição de vulnerabilidade ao bullying ou à vitimização.

\section{DISCUSSÃO}

O objetivo deste estudo foi analisar a qualidade das interações familiares de adolescentes e o envolvimento em situações de bullying escolar. A taxa de prevalência de bullying/ vitimização identificada foi de 10,3\% de estudantes agressores, $10,1 \%$ de vítimas e $5,4 \%$ de vítimas-agressoras. A maioria dos estudantes era oriunda de famílias nucleares. No geral, os resultados confirmaram a associação entre as interações familiares dos adolescentes e o envolvimento em comportamentos de bullying. Especificamente, agressores, vítimas e vítimas-agressoras apresentaram maior pontuação nas dimensões "punição física", "comunicação negativa" e "clima conjugal negativo", quando comparados com os estudantes sem envolvimento com o fenômeno. Essas dimensões negativas foram identificadas como fatores de risco para o bullying ou a vitimização, ao passo que a dimensão "regras e monitoria" se revelou protetiva.

O modelo analítico apresentado representa um passo significativo para a compreensão do bullying, sobretudo quando confirma resultados encontrados em outros estudos, indicando que repetidas experiências no contexto familiar conduzem crianças e adolescentes a internalizar padrões de comportamentos e atitudes que moldarão a conduta social9,16. Essa perspectiva ressalta a importância das experiências no ambiente doméstico para o desenvolvimento individual e social de crianças e adolescentes, bem como na definição de como esse público construirá outras interações no contexto social amplo.

Especificamente, segundo a literatura, os estudantes mais propensos a se envolver em situações de bullying apresentam relações menos favoráveis com seus pais/cuidadores, além de menos sentimentos de envolvimento e empatia familiar ${ }^{6,8,17}$. O bullying também é significativamente associado com menor satisfação em relação à família, maior punição física, conflitos conjugais e resistência dos estudantes à autoridade parental ${ }^{5,11,18,19}$. Por outro lado, os efeitos protetivos do monitoramento e o estabelecimento de regras no contexto familiar são semelhantes aos resultados encontrados em outros estudos, ${ }^{5,20}$. Esse tipo de comportamento apresentado pelos pais ou responsáveis é interpretado como constituinte do envolvimento positivo entre eles e os filhos, aspecto que tem recebido grande destaque na literatura e que é apontado como fator significativo de proteção. O envolvimento também é uma variável caracterizada pela supervisão, estabelecimento de regras, acompanhamento e comunicação positiva ${ }^{8}$.

Assim, os resultados apresentados, consistentes com pesquisas anteriores, explicitam que a dinâmica do bullying não pode ser explicada apenas pelas singularidades ou características pessoais dos estudantes vítimas ou agressores. As evidências apresentadas colocam demandas por iniciativas de enfrentamento para profissionais de diferentes áreas. Nesse sentido, planos de cuidado na área da saúde escolar e saúde do adolescente, a partir de uma leitura ampliada, capaz de integrar diferentes variáveis na compreensão da problemática, são essenciais para o desenvolvimento de uma atenção mais integral e mais potente em termos de enfrentamento desse tipo de violência sofrido por crianças e adolescentes em idade escolar.

É nesse contexto que se estabelecem as implicações para o cuidado na área da saúde, sobretudo na atenção primária, com um caráter inovador na medida em que rompe a lógica assistencialista e preconiza o trabalho direcionado à promoção da saúde e à intersetorialidade ${ }^{21}$. Nesse sentido, as equipes de saúde da Estratégia Saúde da Família (ESF) podem trabalhar com as famílias adscritas às suas áreas de abrangência o fortalecimento de vínculos familiares, estimulando o estabelecimento de regras e monitoria no ambiente doméstico, promovendo padrões de comunicação e clima familiar positivo, que são aspectos protetivos ou que podem minimizar a ocorrência do bullying/vitimização. Essas dimensões podem ser trabalhadas em grupos oferecidos nas unidades de saúde ou, com cada família, no decorrer das visitas domiciliares. Noutra perspectiva, as equipes de saúde podem, preventivamente, auxiliar e orientar pais e cuidadores na identificação de sintomas e queixas relacionadas à vitimização. Para que essas ações sejam efetivadas, os profissionais e as equipes precisam compreender o fenômeno e suas nuances, o que evidencia a importância dos resultados obtidos para a área de estudos.

Noutra perspectiva, o estudo pode ser utilizado para orientar ações relacionadas ao Programa Saúde na Escola que inclui o bullying entre seus temas transversais a serem trabalhados pelas equipes de saúde da atenção primária ${ }^{12}$. O documento que orienta o programa prevê a realização de atividades a serem planejadas e desenvolvidas em cinco componentes, quais sejam: 1) Avaliação clínica e psicossocial; 2) Ações de promoção da saúde e prevenção das doenças e agravos; 3) Educação permanente e capacitação de profissionais da educação e saúde e de jovens; 4) Monitoramento e avaliação da saúde dos estudantes; e 5) Monitoramento e avaliação do Programa 
Saúde na Escola ${ }^{12}$. Essas dimensões, pensadas dentro desse estudo, podem se concretizar da seguinte forma: 1) Identificação de casos de vitimização ou prática de bullying nas escolas, bem como conflitos, violências e práticas inadequadas nas famílias; 2) Ações de orientação das famílias, pais e cuidadores de crianças e adolescentes em idade escolar sobre as consequências do fenômeno para a saúde e o desenvolvimento; 3) Utilização desse estudo em momentos formativos para pensar o impacto das interações familiares no envolvimento dos estudantes em situações de bullying; 4) Acompanhamento de casos identificados de conflitos familiares ou situações de bullying; e 5) Utilização de instrumentos para avaliar periodicamente o clima escolar e a ocorrência de bullying.

Por fim, ressalta-se que os resultados dessa investigação devem ser interpretados considerando suas duas principais limitações do estudo. Primeiramente, a natureza transversal da pesquisa não permite estabelecer nexos causais entre as variáveis em estudo. Em segundo lugar, os instrumentos utilizados na coleta se basearam em relatos retrospectivos de comportamentos de bullying e vitimização, dados que podem ser passíveis de vieses de memória. Para superar essas limitações, estudos longitudinais ou experimentais são necessários, bem como o uso de outras estratégias, tais como a nomeação por pares de vítimas e agressores ou coleta de dados junto a outros informantes (professores, pais, por exemplo).

\section{CONCLUSÕES}

Os pontos fortes desse estudo incluem: 1) A estimativa dos efeitos de variáveis de interação familiar associadas ao bullying escolar entre estudantes brasileiros; 2) O tamanho da amostra de base populacional; e 3) O exame das contribuições das variáveis de interação familiar para o envolvimento de adolescentes em situações de bullying, sendo essa sua maior potência. A validade do estudo assenta, sobretudo, na possibilidade de comparar os resultados obtidos no contexto brasileiro com os resultados obtidos em outros países. Os resultados alcançados ainda sugerem que programas de intervenção devem considerar as variáveis familiares, voltados para a promoção de comportamentos parentais positivos e a redução de fatores negativos nas interações que são associados com aumento de bullying.

\section{CONTRIBUIÇÕES INDIVIDUAIS}

Wanderlei Abadio de Oliveira, Jorge Luiz da Silva, Manoel Antônio dos Santos, Miyeko Hayashida, Simona Carla Silvia Caravita e Marta Angélica lossi Silva - Contribuíram significativamente na concepção e desenho do estudo, na análise e interpretação dos dados; na elaboração do artigo e revisão crítica do seu conteúdo e aprovaram a sua versão final.

\section{CONFLITOS DE INTERESSE}

Não há conflitos de interesse a serem declarados.

\section{AGRADECIMENTOS}

À Fundação de Amparo à Pesquisa do Estado de São Paulo (Processo Fapesp 2014/13062-7) e à Coordenação de Aperfeiçoamento de Pessoal de Nível Superior (Capes) (Bex 7029/14-4) pelo financiamento recebido para o desenvolvimento do estudo.

\section{REFERÊNCIAS}

1. Wu WC, Luu S, Luh DL. Defending behaviors, bullying roles, and their associations with mental health in junior high school students: a population-based study. Bmc Public Health. 2016;16(1):1-10.

2. Oliveira-Menegotto LM, Pasini Al, Levandowski G. 0 bullying escolar no Brasil: uma revisão de artigos científicos. Psicol Teor Prat. 2013;15(2):203-15.

3. Breivik K, Olweus D. An item response theory analysis of the Olweus Bullying Scale. Aggress Behav. 2015;41(1):1-13.

4. Pigozi PL, Machado AL. Bullying na adolescência: visão panorâmica no Brasil. Ciênc Saúde Coletiva. 2015;20:3509-22.

5. Oliveira WA, Silva JL, Sampaio JMC, Silva MAI. Saúde do escolar: uma revisão integrativa sobre família e bullying. Ciênc Saúde Coletiva. 2017;22:1553-64.

6. Yamagata S, Takahashi Y, Ozaki K, Fujisawa KK, Nonaka K, Ando J. Bidirectional influences between maternal parenting and children's peer problems: a longitudinal monozygotic twin difference study. Dev Sci. 2013;16(2):249-59.

7. Shetgiri $R$, Lin $H$, Flores $G$. Trends in risk and protective factors for child bullying perpetration in the United States. Child Psychiatry Hum Dev. 2013;44(1):89-104.

8. Bibou-Nakou I, Tsiantis J, Assimopoulos H, Chatzilambou P. Bullying/victimization from a family perspective: a qualitative study of secondary school students' views. EJPE. 2013;28(1):53-71.

9. Patton DU, Hong JS, Williams AB, Allen-Meares P. A review of research on school bullying among African American youth: an ecological systems analysis. Educ Psychol Rev. $2013 ; 25(2): 245-60$

10. Zottis GA, Salum GA, Isolan LR, Manfro GG, Heldt E. Associations between child disciplinary practices and bullying behavior in adolescents. J Pediatr (Rio J). 2014;90(4):408-14.

11. Tortorelli MFP, Carreiro LRR, Araújo MV. Correlações entre a percepção da violência familiar e 0 relato de violência na escola entre alunos da cidade de São Paulo. Psicol Teor Prat. 2010;12(1):32-42.

12. Brasil. Ministério da Saúde. Departamento de Atenção Básica. Cadernos de Atenção Básica. Brasília: Ministério da Saúde; 2009. v. 24. 96p. (Série B. Textos Básicos de Saúde).

13. Szwarcwald CL, Damacena GN. Amostras complexas em inquéritos populacionais: planejamento e implicações na análise estatística dos dados. Rev Bras Epidemiol. 2008;11:38-45.

14. Cunha JM, Weber LND, Steiner Neto P. Escala de Vitimização e Agressão entre Pares (EVAP). In: Weber LND, Dessen MA, eds. Pesquisando a família - Instrumentos para coleta e análise de dados. Curitiba: Juruá; 2011. p. 103-14.

15. Weber LND, Salvador APV, Brandenburg 0J. Escalas de Qualidade na Interação Familiar. In: Weber LND, Dessen MA, eds. Pesquisando a família - Instrumentos para coleta e análise de dados. Curitiba: Juruá; 2011. p. 61-72. 
16. Ashiabi GS, O'Neal KK. Child social development in context: an examination of some propositions in Bronfenbrenner's Bioecological Theory. Sage Open. 2015;5(2).

17. Moon B, Morash M, McCluskey JD. General strain theory and school bullying: an empirical test in South Korea. Crime Delinquency. 2012;58(6):827-55.

18. Knous-Westfall HM, Ehrensaft MK, MacDonell KW, Cohen P. Parental intimate partner violence, parenting practices, and adolescent peer bullying: a prospective study. I Child Fam Stud. 2012;21(5):754-66.
19. Oliveira WA, Silva MAl, Silva JL, Mello FCM, Prado RR, Malta DC. Associations between the practice of bullying and individual and contextual variables from the aggressors' perspective. J Pediatr (Rio J.). 2016;92(1):32-9.

20. Lösel F, Bender D. Aggressive, delinquent, and violent outcomes of school bullying: do family and individual factors have a protective function? Journal of School Violence. 2014;13(1):59-79.

21. Silva FR, Assis SG. Prevenção da violência escolar: uma revisão da literatura. Educ Pesqui. 2018;44:e157305. 